\title{
Literature Review of Energy Minimization Based Image Segmentation Methods
}

\author{
Pranoti P. Mahakalkar ${ }^{1}$, Dr. Aarti J. Vyavahare ${ }^{2}$ \\ ${ }^{1} \mathrm{P}$ G Student at Department of Electronics \& Tele-communication, \\ Modern College of Engineering, Pune University, Maharashtra, India \\ ${ }^{2}$ Associate professor at Department of Electronics \& Tele-communication, \\ Modern College of Engineering, Pune University, Maharashtra, India
}

\begin{abstract}
The process of image segmentation in computer vision is nothing but extracting or grouping of image objects into various parts. Such extracted parts from image are having information that humans can easily view and separate all objects individually. There is no direct way to recognition such objects using computers, hence we need to have method which can efficiently extract objects from input image for further processing. There are many methods presented for image segmentation in image processing domain. Such segmentation methods are based on different image features. In this paper we are focusing on field of energy minimization as it applies to image segmentation. The traditional methods for image segmentation are unreliable, noise insertion, shape variation. Therefore to address this limitations and achieves better image segmentation since from last decade energy minimization based methods introduced by various researchers due to their characteristic of considering various segmentation goals. In general, process of energy minimization methods is based on evaluating the segmentation goodness for input image, and then further minimization of evaluated function resulted into image segmentation. In this paper we are presented the survey on different energy minimization based image segmentation methods with their advantages and disadvantages. Basically all this methods falls under the category of level set function based methods.
\end{abstract}

Keywords: Image Segmentation, Image processing, Energy Minimization, Level Set Methods, Region based, Edge based, Minimizer

\section{Introduction}

In computer image processing, digital image is given input to computerized application in order to process using signal processing concepts and resulted into the output which can be either set of image features, set of parameters related to image or an image. The object recognition as well as computer vision which is widely used in many real time applications like processing and analysis of medical image, target tracking, image retrieval etc. using the concepts of image processing.[1]Image processing method can process the $2 \mathrm{D}$ or $3 \mathrm{D}$ images using computer. Generally image is nothing but the method of two coordinates $\mathrm{x}$ and $\mathrm{y}$ with the brightness at these variables. Image processing is composed of three main parts image processing, image segmentation, and analysis. For image processing, segmentation of image is most important. Image segmentation is an important part in image processing. In image segmentation automated approach is used to segment input image into different regions with meaningful information. The basic objective of image segmentation is to simplify the image representation into the group of objects those are having more meaningful information and hence further easy for analysis. Image segmentation basically used for describing boundaries as well as objects from input images which is done by assigning label to each image pixel of input image. All pixels with same label are referred as similar visual characteristics. This process of image segmentation is widely used in real time applications such as Tumor detection, other medical image processing, tissue volume measurement, planning of treatment, objects tracking in satellite imagine, face recognition, fingerprint recognition, surgery etc. From raw image basic structure of image is extracted. In medical image processing, this process helps for detecting the part of Tumor or extracting white region from the gray region from brain scan image. Therefore such process of segmentation becomes vital part in domain of medical image processing which helps to detect and visualize different kinds of diseases. [2]

There are number of segmentation methods presented since from last two decades. But recent methods are based on energy minimization based image segmentation. Partial differential equation (PDE) is method for image segmentation. The segmentation of image is done by solving equations of PDE through the numerical methods. In this category, curve propagation is most famous method under the PDE and used in applications like object tracking, object extraction, stereo reconstruction, satellite imagine etc.[3]The main idea of this method is to evaluate the initial curve towards cost function minimum potential. Apart from this many other methods presented under the domain of PDE equations. Parametric method is one of the PDE equations based method in which parameterization of counters is done as per sampling strategy set as well as elements evolving is done as per the internal terms and image. Another method called fast marching presented for image segmentation. Further this method was improved by allowing both positive as well as negative propagation speed. This improved method for image segmentation is referred as generalized fast marching technique.

After this, level set methods presented for image segmentation under this domain. These methods initially proposed by authors Osher and Sethian. At the time of their introduction, such methods are accepted majorly for capturing of dynamic shapes as well as interfaces. Level set function based methods basically work by setting the level set function in order to represent contour initially at zero level set of function of higher dimensional set as well as motion formulation of contour as evolution of function of level set. [4, 5]Models of level set can describe the 


\section{International Journal of Science and Research (IJSR) \\ ISSN (Online): 2319-7064 \\ Index Copernicus Value (2013): 6.14 | Impact Factor (2014): 5.611}

target object through change in complex topology based on various functions of energy generated from input image. In parametric active counter models, the tasks like splitting and merging are difficult; hence this can be achieved by level set methods. The main challenging task with level set methods is that segmentation energy function definition. Minimization of energy function is done while evolving counter process is going based on edge energy through region energy or boundary detector based on the regiondescriptor. Level set methods are divided into two main categories such as edge based method and region based methods according to the energy function definition.

There are many methods presented fewer than two categories of level set methods, however they suffered from limitations. The existing level set methods are having disadvantage while producing the efficient segmentation without prior information over image segments and in noisy images. To overcome these limitations recently energy model proposed. In this paper our aim is to present and discuss different existing methods of image segmentation using minimum energy function with their advantages and disadvantages. Below in section II, discussing different types of level set methods. In section III, survey over different image segmentation methods presented our main focus on level set methods. Finally section IV, conclusion and future work discussed. [6]

\section{Level Set Methods}

As we discussed earlier, there are two types of level set image segmentation methods such as edge based models as well as region based models.

\subsection{Edge-Based Models}

In these methods, if the evolving counter speed reaches to zero, then contour stopping on specific boundary when the speed of the evolving contour becomes zero, the contour stops on the desired boundary with the major gradients. The famous example of such methods is geodesic active contour or model of geodesic level set. Energy function minimization is the length of contours geodesic in this model, especially from boundaries. As such models are uses only edge characteristics in order to locate target objects, these level set models are sensitive to image noise and initial conditions. Frequently these models are suffering from the problems of serious boundary leakage in images along with boundaries of weak object.[7]

\subsection{Region-Based Models}

These are another category level set models which are introduced by the author Ronfard et al. in order to identify every ROI (region of interest) with help of region descriptor for guiding level set function motion. At first basic piecewise smooth (PS) equation was introduced for image segmentation under this category. After that PS level set methods introduced to overcome disadvantages of weak region or blurred region boundaries as well as considering the assumption of intensity homogeneity for every disjoint region in input image. The method presented under this category called Chan-Vese (CV) model is example of piecewise constant (PC) model. This method is capable of doing image segmentation into the background and foreground using two piecewise constant values. Further if it is required to segment image into more than two segments as well as different regions, then authors Vese and Chan and Tsai et al. introduced two same methods. These methods were based on multiphase level set techniques in order to partition images into multiple segments. By using CV model, further global statistical energy function proposed by Roussos and Deriche to evolve function of level set. [8]

Such region based methods used to segment image into number of regions specified by user. But for accurate segmentation, it is challenging task to define number of image segments before segmentation process. Incorrect numbers of segments would lead to an incorrect segmentation.

\section{Literature Review}

The task of image segmentation is to find a collection of non-overlapping sub-regions of a given image. There are many other obvious applications. Mathematically, given an image u: $\Omega$ C R2 (or R3) $\rightarrow \mathrm{R}+$, we want to find closed sets $\Omega$ i satisfying

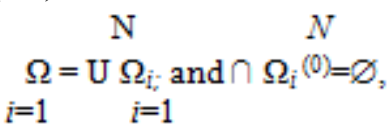

Such that $\mathrm{F}(\mathrm{u} ; \Omega \mathrm{i})=0$; where $\mathrm{F}$ is some functional that defines the segmentation goals. Here, $\Omega$ i (0) denotes the interior of $\Omega \mathrm{i}$ : As in the example of finding tumours, typically, $\mathrm{N}$ is taken to be 2 (sometimes $\mathrm{N}=3$ ), and $\Omega 1$ is taken to be the region corresponding to the Tumor, while $\Omega 2$ contains everything else. It is then natural to devise a level set method to perform this task, by representing, for example, $\Omega 1$ as the region in which $\phi$ is non-negative. A slightly more general statement would be to perform segmentation from a given set of images $u j$ that come from different sources. For example, one might be interested to segment stealth fighter jets from both the conventional radar signals and also the infrared images. $[13,14]$

Very often, the definition of what belongs to the "desired" regions depends on the gray scale intensity of the given image, and the problem of finding such regions is formulated as a variation problem; i.e. the solution minimizes some "energy". In a standard level set method, $\phi$ is used to represent $\Omega \mathrm{i}$ and $\partial \Omega \mathrm{i}$. This is the setting of our discussion. In this section, we describe some level set segmentation methods based on this type of definition.

\subsection{Variation Level Set Method}

Assume that the energy functional $\mathrm{E}$ is an integral operator on $u$ over $\Omega 0$ :

(3.1.1) $\mathrm{E}(\mathrm{u} ; \Omega 0)=\int \Omega 0 \mathrm{~F}(\mathrm{u}(\mathrm{x})) \mathrm{dx}$; 


\section{International Journal of Science and Research (IJSR) \\ ISSN (Online): 2319-7064 \\ Index Copernicus Value (2013): 6.14 | Impact Factor (2014): 5.611}

and the non-positive region of $\phi$ defines $\Omega 0$; i.e. $\{\phi \leq 0\}=$ $\Omega 0$. The key idea of the variation level set method formulated in is that the above integral can be written as

(3.1.2) $\int \Omega F(u(x)) d x=\int R 2 \chi \Omega(x) F(u(x)) d x=\int R 2 H(-$ ф) $\mathrm{F}(\mathrm{u}) \mathrm{dx}$,

Where $\mathrm{H}$ is the HEaviside function: $\mathrm{H}(\mathrm{x})=1 \mathrm{i} \mathrm{f} \times 0$ and $\mathrm{H}(\mathrm{x})=0$ elsewhere. One can then try to nd the minimizer $\phi$ for this energy. [15]

\subsection{The Chan-Vese Algorithm}

This is closely related to the classical Mumford-Shah algorithm [16], but uses a simple level set framework for its implementation. We present the original Chan-Vese segmentation algorithm, and discuss various aspects of this algorithm.

\subsubsection{Basic formulation. The minimization problem is:} $\min _{\Phi \in B V(\Omega), c 1, c 2 \epsilon R^{+}} E(\Phi, c 1, c 2 ; u 0)$,

Where the energy is defined as

$\mathrm{E}(\Phi, \mathrm{c} 1, \mathrm{c} 2: \mathrm{u} 0)=\mu \int_{-} \Omega \quad \delta(\Phi)\left|\nabla \_\Phi \quad\right| \mathrm{dx}+\lambda \_1 \quad \int_{-} \Omega \quad|\mathrm{u} 0-\mathrm{c} 1|^{\wedge} 2$ $\mathrm{H}(\Phi) \mathrm{dx}+\lambda \_2 \int \Omega|\mathrm{u} 0-\mathrm{c} 2|^{\wedge} 2(1-\mathrm{H}(\Phi)) \mathrm{dx}$.

If one regularizes the $\delta$ function and the Heaviside function by two suitable smooth functions $\delta \epsilon$ and $H \epsilon$, then formally, the Euler-Lagrange equations can be written as

$$
\begin{gathered}
\partial_{\Phi} \mathrm{E}=-\delta \epsilon(\phi)\left[\mu \nabla \cdot \frac{\nabla(\Phi)}{|\nabla \Phi|}-v-\lambda_{1}\left(u_{0}-c_{1}\right)^{2}\right. \\
\left.+\lambda_{2}\left(u_{0}-c_{2}\right)^{2}\right]=0,
\end{gathered}
$$

With natural boundary condition

$$
\text { (3.2.3) }
$$

$$
\frac{\delta \epsilon(\Phi)}{|\nabla \Phi|} \frac{\partial \Phi}{\partial \underset{n}{\rightarrow}}=0 \text { on } \partial \Omega \text {. }
$$

$C_{1}(\Phi)=\frac{\int \Omega^{\mu 0(x) H \varepsilon(\Phi(x)) d x}}{\int \Omega^{(H \varepsilon(\Phi(x))) d x}}$,

and

$C_{1}(\Phi)=\frac{\int \Omega^{\mu 0(x) H \varepsilon(\Phi(x)) d x}}{\int \Omega^{(H \varepsilon(\Phi(x))) d x}}$,
3.2.2. Discretization. A common approach to solve the minimization problem is to perform gradient descent on the regularized Euler-Lagrange equation (3.2); i.e. solving the following time dependent equation to steady state:

(3.2.2.1)

$$
\begin{aligned}
& \frac{\partial \Phi}{\partial t}=-\partial_{\Phi} \mathrm{E} \\
& \partial_{\Phi} \mathrm{E}=-\delta \epsilon(\phi)\left[\mu \nabla \cdot \frac{\nabla(\Phi)}{|\nabla \Phi|}-v-\lambda_{1}\left(u_{0}-c_{1}\right)^{2}\right. \\
& \left.+\lambda_{2}\left(u_{0}-c_{2}\right)^{2}\right]=0 \text {, }
\end{aligned}
$$

Here, we remind the readers that $\mathrm{c} 1(\Phi)$ and $\mathrm{c} 2(\Phi)$ are defined in (3.3) and (3.4).

In Chan-Vese algorithm, the authors regularized the Heaviside function used in (3.3) and (3.4):

$H_{2, \varepsilon}(z)=\frac{1}{2}\left(1+\frac{2}{\pi} \arctan \left(\frac{z}{\varepsilon}\right)\right)$

and de ne the delta function as the derivative of it:

$H_{2, \varepsilon}(z)=\frac{1}{2}\left(1+\frac{2}{\pi} \arctan \left(\frac{z}{\varepsilon}\right)\right)$

Equation (3.5) is then discretized by a semi-implicit scheme; i.e. to advance from $\Phi \mathrm{Ni} ; \mathrm{j}$ to $\Phi \mathrm{ni} ;+\mathrm{j} 1$, the curvature term right hand side of (3.5) is discretized as described in the previous section using the value of $\Phi \mathrm{ni} ; \mathrm{j}$ , except for the diagonal term $\Phi \mathrm{i} ; \mathrm{j}$, which uses the implicitly de ned $\Phi$ ni; $+\mathrm{j} 1$. The integrals de ning $\mathrm{c} 1$ ( $\Phi)$ and c2 $(\Phi)$ are approximated by simple Riemann sum with the regularized Heaviside function de Ned above. $\Phi$ t is discretized by the forward Euler method: $(\Phi \mathrm{Ni} ;+\mathrm{j} 1 \Phi \mathrm{Ni}$; j) $=\Delta \mathrm{t}$. Therefore, the nel update formula can be conceptually written as [17]

$\Phi_{i, j}^{n+1}=\frac{1}{1+\alpha_{k}}\left(\Phi_{i, j}^{n}+G\left(\Phi_{i-1, j, \Phi_{i+1, j}^{n}}^{n}, \Phi_{i, j-1}^{n}, \Phi_{i, j+1}^{n}\right)\right)$ 


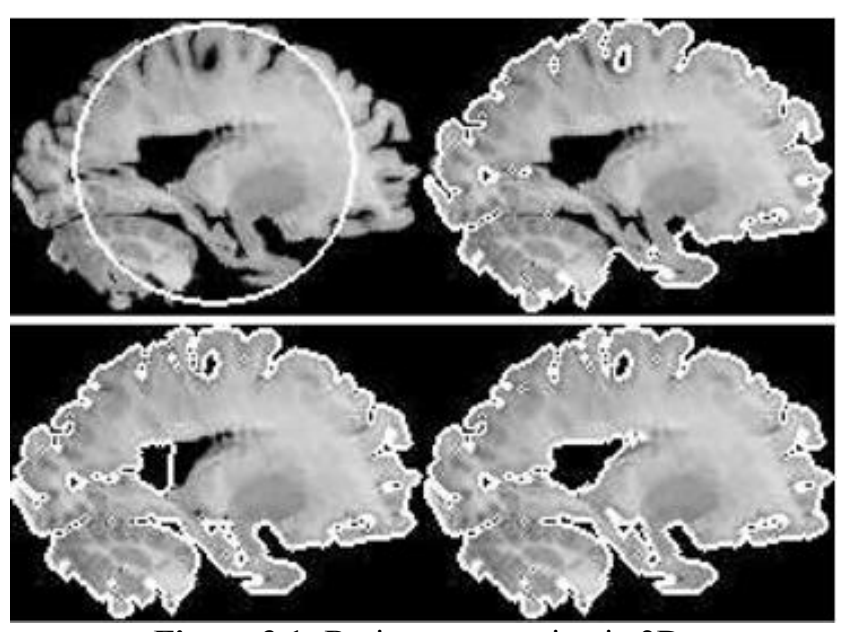

Figure 3.1: Brain segmentation in 2D

\subsection{Fast one-Pass Segmentation Algorithms.}

Recently, Gibou and Fedkiw, and Song and Chan, proposed some fast methods that are based on the ChanVese level set segmentation formulation. These algorithms are built upon ipping the values of $\Phi$ at each grid point/pixel from positive to negative or vice versa according to a rule $\mathrm{R}$, and contain 4 main steps: [18]

Initialize $\Phi 0: \Omega \rightarrow\{-1,1\}$

Advance: for each grid point, set $\Phi \mathrm{n}+1(\mathrm{x})=\Phi \mathrm{n}(\mathrm{x})$ if $\mathrm{R}$ $(\Phi \mathrm{n}+1 ; \Phi \mathrm{n} ; \mathrm{x})=1$ : (Perform regularization if needed.)

Repeat until $\Phi \mathrm{n}+1 \Phi \mathrm{n}$.

For example, in Gibou and Fedkiw's algorithm, $\mathrm{R}(\Phi \mathrm{n}+1$; $\Phi \mathrm{n})=1$ if $\mathrm{V}(\mathrm{FN}) \operatorname{sign}(\Phi \mathrm{n})<0$;

Here $\mathrm{V}$ corresponds to the tting term in the Euler-Lagrange equation:

$\mathrm{V}(\Phi \mathrm{n} ; \mathrm{x}):=\lambda 1(\mathrm{u} 0-\mathrm{c} 1(\Phi \mathrm{n})) 2+\square 2(\mathrm{u} 0-\mathrm{c} 2(\Phi \mathrm{n})) 2:$

$\mathrm{E}(\Phi \mathrm{n}+1 ; \mathrm{c} 1 ; \mathrm{c} 2 ; \mathrm{u} 0) \mathrm{E}(\Phi \mathrm{n} ; \mathrm{c} 1 ; \mathrm{c} 2 ; \mathrm{u} 0)$

Hence, the sign of $\Phi \mathrm{n}(\mathrm{x})$ is ipped only if the energy (3.1) is non-increasing. This provides stability of the algorithm when compared to Gibou and Fedkiw's in which there is no checking on the energy descent, at the cost of some speed of implementation. [19]

We remark that there is a close connection between these two "level set" based methods to the "T-convergence" base methods.

The Chan-Vese segmentation method can be approximated by the following variation problem:

\section{(3.3.2)}

$\mathrm{E} \epsilon(\mathrm{u} ; \mathrm{c} 1 ; \mathrm{c} 2 ; \mathrm{u} 0):=\mu \int_{\varepsilon} \llbracket|\nabla \mathrm{u}| \rrbracket \wedge 2+1 / \varepsilon \mathrm{W}(\mathrm{u}) \mathrm{dx}+\lambda 1 \int \mathrm{u}^{\wedge} 2$ $\llbracket\left(u \_0-c \_1\right) \rrbracket \wedge 2+\lambda 2 \int \llbracket(1-u) \rrbracket \wedge 2$ \(u_o-c_2 $) \rrbracket \wedge 2 d x$;

\subsection{Segmentation of Multiple "Phases"}

There are efforts to generalize the level set methods for multiphase computation. [14] For example, in, each partition $\Omega i$ is represented by a level set function i. It is then important to enforce the constraints that 1) the regions represented do not $\operatorname{overlap}\left(\bigcap_{-}(\mathrm{i}=1)^{\wedge} \mathrm{N} \rrbracket\left\{\Phi \_\mathrm{i} \rrbracket<0\right)\right.$, and 2 ) there are no unclaimed regions; i.e. every point in $\Omega$ belongs to certain $\left(\Omega \quad \mathrm{i} U_{-}(\mathrm{i}=1)^{\wedge} \mathrm{N} \llbracket\left\{\Phi \_\mathrm{i} \leq 0\right\}\right) \rrbracket$. Interesting formulae are derived in the variation setting to enforce these two conditions. However, this approach may be expensive when the number of phases is large. [20]

In, the authors use the sign of the level set functions $\Phi \mathrm{j}$ as a binary coding for the phases, each assigned a nonnegative integer value. Suppose there are four Phases, $\Omega \mathrm{i}, \mathrm{i}$ $=, 3$, and two level set functions $\Phi 0$ and $\Phi 1$ are used for their representation. One can then write, for instance,

$$
\begin{aligned}
& \Omega_{-} 0=\left\{\Phi \_0 \geq 0\right\} \cap\left\{\Phi \_1 \geq 0\right\}, \\
& \Omega_{-} 1=\left\{\Phi \_0 \leq 0\right\} \cap\left\{\Phi \_1 \geq 0\right\}, \\
& \Omega_{-} 2=\left\{\Phi \_0 \geq 0\right\} \cap\left\{\Phi \_1 \leq 0\right\}, \\
& \Omega_{-} 3=\left\{\Phi \_0 \leq 0\right\} \cap\left\{\Phi_{-} 1 \leq 0\right\} .
\end{aligned}
$$

To full generality, write the phase number $\mathrm{i}$ in binary format $=\sum_{-}(\mathrm{k}=0)^{\wedge}(\mathrm{n}-1)$ 带 『c_k. $2^{\wedge} \mathrm{k} \rrbracket$, where ck takes on either 0 or 1 . Then one way of using $\left\{\Phi \_\mathrm{i}\right\}(\mathrm{n}-1)$ ( $\mathrm{k}=0$ ) level set functions to represent $\square \mathrm{i}$ is to identify (3.4.2)

$\Omega_{i}=\bigcap_{k=0}^{n-1}\left\{x \in \Omega:\left(1-c_{k}\right) . \Phi_{k}(x) \geq 0\right\}$.

A drawback of this approach is the potential misidentify cation of what is sup-posed to be categorized as one single phase to two or more "different" phases, since the formulation really comes with $2 \mathrm{n}$ phases with $\mathrm{n}$ level set functions.[17,18]

\section{Conclusion and Future Work}

In this paper we summarized background of image segmentation, fundamentals, definitions, and methods, advantages of methods and disadvantages of methods. Our aim was to present review of different energy minimization based image segmentation techniques. Image segmentation is widely used process in many of real time applications for tracking, identification and analysis. We focused methods under the category of level set functions. Level set methods basically used in order to solve the problems of propagation of surfaces or curve implicitly. Most of methods presented in this paper are from level set methods only due to its various advantages over traditional image segmentation methods. Through the paper we presented the different energy minimization techniques used for image segmentation, different blocks of image segmentation etc. For future work, this domain is still open for improvement to achieve most accurate and minimum energy function based image segmentation method. In addition to this, in future practical evaluation of currently 


\section{International Journal of Science and Research (IJSR) \\ ISSN (Online): 2319-7064}

Index Copernicus Value (2013): 6.14 | Impact Factor (2014): 5.611

presented efficient methods should be done with extensive comparative analysis.

\section{References}

[1] C. H. Chen, T. Y. Chen, D. J. Wang, and T. J. Chen, "A cost-effective people-counter for a crowd of moving people based on two-stage segmentation," J. Inf. Hiding Multimedia Signal Process. vol. 3, no. 1, pp. 12-23, Jan. 2012.

[2] F. C. Chang and H. M. Hang, "A relevance feedback image retrieval scheme using multi instance and pseudo image concepts," IEICE Trans. Inf. Syst., vol. 89, no. 5, pp. 1720-1731, May 2006.

[3] F. C. Chang, H. M. Hang, and H. C. Huang, "Research friendly MPEG- 7 software testbed," in Proc. Video Commun. Process. Conf. Symp. Electron. Imaging Sci. Technol., Jun. 2003, pp. 890-901.

[4] G. Malathi and V. Shanthi, "Statistical measurement of ultrasound placenta images complicated by gestational diabetes mellitus using segmentation approach," J. Inf. Hiding Multimedia Signal Process. vol. 2, no. 4, pp. 332-343, Oct. 2011.

[5] J. A. Noble and D. Boukerroui, "Ultrasound image segmentation: A survey," IEEE Trans. Med. Imaging, vol. 25, no. 8, pp. 987-1010, Aug. 2006.

[6] S. Osher and J. Sethian, "Fronts propagating with curvature-dependent speed: Algorithms based on Hamilton-Jacobi formulations," J. Compute. Phys., vol. 79, no. 1, pp. 12-49, Nov. 1988.

[7] D. Cremers, M. Rousson, and R. Deriche, "A review of statistical approaches to level set segmentation: Integrating colour, texture, motion and shape," Int. J. Compute. Vis., vol. 72, no. 2, pp. 195-215, 2007.

[8] C. Li, C. Xu, C. Gui, and M. D. Fox, "Distance regularized level set evolution and its application to image segmentation," IEEE Trans. Image Process., vol. 19, no. 12, pp. 3243-3254, Dec. 2010.

[9] V. Caselles, R. Kimmel, and G. Sapiro, "Geodesic active contours," Int. J. Compute. Vis., vol. 22, no. 1, pp. 61-79, 1997.

[10] V. Caselles, F. Catté, T. Coll, and F. Dibos, "A geometric model for active contours in image processing," Numer. Math., vol. 66, no. 1, pp. 1-31, 1993.

[11]A. Yezzi, Jr., S. Kichenassamy, A. Kumar, P. Olver, and A. Tannenbaum, "A geometric snake model for segmentation of medical imagery," IEEE Trans. Med. Imag., vol. 16, no. 2, pp. 199-209, Apr. 1997.

[12] C. Li, C.-Y. Kao, J. C. Gore, and Z. Ding, "Minimization of region scalable fitting energy for image segmentation," IEEE Trans. Image Process., vol. 17, no. 10, pp. 1940-1949, Oct. 2008.

[13]F. Alvarez, F. Guichard, J.-M. Morel, and P.-L. Lions. Axioms and fundamental equations of image processing. Arch. Rat. Mech. and Analyse. 123:199257,

[14] Tony F. Chan and Laminate A. Vese. Active contours without edges. IEEE Transactions on Image Processing, 10(2):266-277, 2001

[15] Antonio Marquita and Stanley Osher. Explicit algorithms for a new time dependent model based on level set motion for nonlinear deburring and noise removal. SIAM J. Sci. Compute, 22(2):387-405 (electronic), 2000.

[16]David Mumford and Jayant Shah. Optimal approximations by piecewise smooth functions and associated variation problems. Comm. Pure Appl. Math., 42(5):577-685, 1989.

[17] Stanley Osher and Barry Merriman. The Wolff shape as the asymptotic limit of a growing crystalline interface. Asian J. Math., 1(3):560-571, 1997.

[18] Stanley Osher and Selim Esedoglu. Decomposition of images by the anisotropic rudin-osherfatemi model. UCLA CAM Report, 03(34), 2003.

[19] Danping Peng, Stanley Osher, Barry Merriman, and Hong-Kai Zhao. The geometry of Wulff crystal shapes and its relations with Riemann problems. In Nonlinear partial differential equations (Evanston, IL, 1998), pages 251-303. Amer. Math. Soc., Providence, RI, 1999.

[20] Luminita A. Vese and Stanley J. Osher. Modelling textures with total variation minimization and oscillating patterns in image processing. UCLA CAM Report, 02(20), 2002 